\title{
Cesarean Section on the Risk of Celiac Disease in the Offspring: The Teddy Study
}

\author{
${ }^{*}$ Sibylle Koletzko, ${ }^{\dagger}$ Hye-Seung Lee, ${ }^{\ddagger}$ Andreas Beyerlein, ${ }^{\S}$ Carin A. Aronsson, ${ }^{\ddagger}$ Michael Hummel, \\ ${ }^{\|}$Edwin Liu, ${ }^{\circledR}$ Ville Simell, ${ }^{*}$ Kalle Kurppa, ${ }^{\S}$ A ke Lernmark, ${ }^{* *}$ William Hagopian, ${ }^{\dagger \dagger}$ Marian Rewers, \\ ${ }^{\ddagger}$ Jin-Xiong She, ${ }^{\S \S|\||}$ Olli Simell, ${ }^{\S \S \uparrow}$ Jorma Toppari, ${ }^{\ddagger}$ Anette-G. Ziegler, ${ }^{\dagger}$ Jeffrey Krischer, \\ and ${ }^{\S}$ Daniel Agardh, for the TEDDY Study Group
}

\begin{abstract}
Objective: Cesarean section (C-section) is associated with various immunemediated diseases in the offspring. We investigated the relationship between mode of delivery and celiac disease (CD) and CD autoimmunity (CDA) in a multinational birth cohort.

Methods: From 2004 to 2010, infants from the general population who tested positive for HLA DR3-DQ2 or DR4-DQ8 were enrolled in The Environmental Determinants for Diabetes in the Young (TEDDY) study. Children were annually screened for transglutaminase autoantibodies, if positive, they are retested after 3 to 6 months and those persistently positive defined as CDA. Associations of C-section with maternal (age, education level, parity, prepregnancy weight, diabetes, smoking, weight gain during pregnancy) and child characteristics (gestational age, birth weight) were examined by Fisher exact test or Wilcoxon rank-sum test. Hazard ratios (HRs) for CDA or CD were calculated by Cox proportional hazard regression models.

Results: Of 6087 analyzed singletons, 1600 (26\%) were born by C-section (Germany 38\%, United States 37\%, Finland 18\%, Sweden 16\%), and the remaining were born vaginally without instrumental support; $979(16 \%)$ had developed CDA and $343(6 \%)$ developed CD. C-section was associated with lower risk for CDA (hazard ratio $[\mathrm{HR}]=0.85 ; 95 \%$ confidence interval $[\mathrm{CI}]$ $0.73,0.99 P=0.032)$ and $\mathrm{CD}(\mathrm{HR}=0.75 ; 95 \% \mathrm{CI} 0.58,0.98 ; P=0.034)$. After adjusting for country, sex, HLA-genotype, CD in family, maternal education, and breast-feeding duration, significance was lost for CDA $(\mathrm{HR}=0.91 ; 95 \%$ CI $0.78,1.06 ; P=0.20)$ and $\mathrm{CD}(\mathrm{HR}=0.85 ; 95 \% \mathrm{CI}$ $0.65,1.11 ; P=0.24)$. Presurgical ruptured membranes had no influence on CDA or CD development.

Conclusion: C-section is not associated with increased risk for CDA or CD in the offspring.
\end{abstract}

Key Words: celiac disease autoimmunity, mode of delivery, screening, tissue transglutaminase, vaginal delivery

(JPGN 2018;66: 417-424)

Received March 18, 2017; accepted June 3, 2017.

From the *Dr. v. Hauner Children's Hospital, University Munich Medical Center, Munich, Germany, the $†$ Health Informatics Institute, Department of Paediatrics, Morsani College of Medicine, University of South Florida, Tampa, FL, the łInstitute of Diabetes Research, Helmholtz Zentrum München, Klinikum rechts der Isar, Technische Universität München, and Forschergruppe Diabetes e.V., Neuherberg, Germany, the §Department of Clinical Sciences, Lund University, Skane University Hosptial, Malmo, Sweden, the $\|$ Digestive Health Institute, University of Colorado, Children's Hospital Colorado, Aurora, CO, the $₫$ Medicity Laboratory, University of Turku, Turku, the \#Tampere Centre for Child Health Research, University of Tampere and Tampere University Hospital, Tampere, Finland, the $* *$ Pacific Northwest Diabetes Research Institute, Seattle WA, the ††Barbara Davis Center for Childhood Diabetes, University of Colorado, Aurora CO, the $\$$ †enter for Biotechnology and Genomic Medicine, Medical College of Georgia, Augusta University, Augusta, GA, the $\S \S$ Department of Pediatrics, Turku University

\section{What Is Known}

- Cesarean sections and celiac disease are both increasing worldwide.

- Meta-analyses on mode of delivery have reported cesarean section to be associated with an increased risk of chronic inflammatory disorders in the offspring.

- Results are conflicting with respect to risk of later celiac disease if born by cesarean section compared to vaginal delivery, but also controversial regarding the later diagnosis of celiac disease after planned versus emergency cesarean section.

\section{What Is New}

- Results of this multinational prospective screening study including $>6000$ infants recruited from the general population with known HLA risk genotypes for celiac disease show that cesarean section is not associated with an increased risk for celiac disease autoimmunity or celiac disease.

- Our analysis emphasizes the importance of considering confounders known to be associated with mode of delivery.

- Environmental factors during later infancy or early childhood are more likely responsible for the increasing incidence of $C D$.
Hospital, the |||| Research Centre of Applied and Preventive Cardiovas-

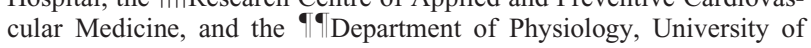
Turku, Turku, Finland.

Address correspondence and reprint requests to Sibylle Koletzko, MD, Dr. v. Hauner Children's Hospital, University Munich Medical Center, Lindwurmstr. 4, 80337 Munich, Germany (e-mail: sibylle.koletzko@med.uni-muenchen.de).

Members of the TEDDY Study Group are listed in a Supplemental Data File.

Funding Source: National Institute of Health (NIH), Department of Health and Human Services (DHHS), the National Institute of Diabetes and Digestive and Kidney Diseases (NIDDK), National Institute of Allergy and Infectious Disease (NIAID), National Institute of Environmental Health Sciences (NIEHS), National Institute of Child Health and Human Development (NICHD), Juvenile Diabetes Research Foundation (JDRF), Centers for Disease Control and Prevention (CDC). 
he increasing rates of cesarean section (C-section) worldwide have provoked considerable public health interest for the short and long-term effects in infants and children (1). Reported short-term negative health effects during the neonatal period include impaired lung function, hypoglycemia, reduced breastfeeding initiation, and altered innate and adaptive immune responses (1). Reported long-term effects include an increased risk of immune-mediated conditions such as asthma and other allergic diseases, systemic connective tissue disorders, juvenile arthritis, and type 1 diabetes mellitus (T1D) (2-4). Results from animal models suggest that the microbiota plays a pivotal role in shaping the innate and adaptive immune response, and is also important in human health and disease (5). Mode of delivery is crucial for the acquisition of the microbiota after birth (6). Infants born naturally or by $\mathrm{C}$-section after rupture of the membranes are exposed to the maternal vaginal flora during birth, whereas infants born by planned $\mathrm{C}$-section are not. Other factors such as perinatal antibiotic use by the mother or the child or the type of feeding (breast-feeding, formula feeding, and solid food introduction) will further influence the composition of the microbiome.

Celiac disease (CD) is an immune-mediated systemic autoimmune disease elicited by gluten in genetically susceptible individuals carrying the HLA-DQ2 and/or DQ8 haplotypes. It is characterized by the presence of circulating tissue transglutaminase autoantibodies (tTGAs) and an inflammatory enteropathy with villous atrophy (7). CD affects approximately $1 \%$ to $2 \%$ of the population in Europe and North America with increasing incidence during the past 2 decades (8). Although positivity for HLA-DQ2, DQ8, or both haplotypes is a prerequisite for disease, only a minority of individuals at genetic risk is affected in spite of the fact that gluten-containing foods are globally consumed. In addition, the overall contribution of known genes to the risk of CD account for less than half of all heritability (9). This strongly indicates that other environmental factors are contributing to the increase in prevalence of $\mathrm{CD}$ in the general population $(8,10)$.

It has been hypothesized that infants born by $\mathrm{C}$-section acquire different bacterial communities compared to vaginally delivered infants (6), which may influence the short- and long-term immune responses to environmental factors, thereby predisposing to autoimmunity (5). Data from previous studies of C-section on the risk of CD in offspring, however, are conflicting, showing a positive association with $\mathrm{CD}(11-14)$ or no correlation in epidemiological studies $(4,15,16)$. The aim of the present analysis was therefore to evaluate in a large population-based cohort whether C-section is associated with an increased risk for tTGA positivity and CD after adjusting for known confounders for the disease (17) and for maternal and child characteristics, which are possibly related to the risk of having a $\mathrm{C}$-section.

\section{METHODS}

\section{Study Design and Participants}

Data were analyzed from the ongoing The Environmental Determinants of Diabetes in the Young (TEDDY) study. TEDDY is a multicenter observational cohort study with the aim of identifying environmental factors associated with T1D and CD in children with an HLA-conferred risk prospectively followed from birth (18). Between September 2004 and February 2010, 424,788 newborns were screened for HLA-DR-DQ genotypes, and 21,589 were identified as carriers of one of the HLA genotypes targeted in TEDDY (19). Of those, 8676 infants were enrolled from families with a first-degree relative with T1D $(\mathrm{n}=951)$ and from families without $(\mathrm{n}=7725)$ in 6 centers located in Colorado, Georgia, and Washington in the United States, and in Finland, Germany, and Sweden. Written informed consent was obtained for all study participants. The study was approved by local institutional review boards and is monitored by an External Advisory Board formed by the National Institutes of Health.

We analyzed the singleton TEDDY children screened for tTGA with information on mode of delivery. Excluded from the current analysis were participants born by instrumental support (vacuum, forceps) owing to a higher likelihood of having postpartum complications including infections, need for intensive care or hospitalization, and antibiotics.

\section{Outcome: Celiac Disease Autoimmunity and Celiac Disease}

Participants were annually screened for tTGA using a radioligand binding assay as previously described (20). Annual screening started at 2 years of age. tTGA-positive children $(\geq 1.3$ $\mathrm{U})$ were retested after 3 months, or after 6 months if the first positive sample occurred after 48 months (21). Children who were tTGA-positive in 2 consecutive samples were defined as having CDA and were referred back to their physician for further evaluation of possible $\mathrm{CD}$. The decision to perform biopsies was unaffected by the study. Multiple biopsies from different parts of the duodenum were recommended, with histologic scoring according to the modified Marsh classification (22). A Marsh score $\geq 2$ was defined as biopsy-proven CD (7). For children with tTGA positivity, their serum samples collected before the first positive sample were tested for tTGA to find the earliest age of seroconversion (21). For those children without a Marsh score, a mean tTGA level of 2 consecutive samples $>100 \mathrm{U}$ was also considered as having CD (21). This threshold was selected based on an internal review of all biopsied individuals to achieve $\geq 95 \%$ specificity (17).
Financial support: The TEDDY study is supported by the following grants: U01 DK63829, U01 DK63861, U01 DK63821, U01 DK63865, U01 DK63863, U01 DK63836, U01 DK63790, UC4 DK63829, UC4 DK63861, UC4 DK63821, UC4 DK63865, UC4 DK63863, UC4 DK63836, UC4 DK95300, UC4 DK100238, UC4 DK106955, and Contract No. HHSN267200700014C from the National Institute of Diabetes and Digestive and Kidney Diseases (NIDDK), National Institute of Allergy and Infectious Diseases (NIAID), National Institute of Child Health and Human Development (NICHD), National Institute of Environmental Health Sciences (NIEHS), Juvenile Diabetes Research Foundation (JDRF), and Centers for Disease Control and Prevention (CDC). This work supported in part by the
NIH/NCATS Clinical and Translational Science Awards to the University of Florida (UL1 TR000064) and the University of Colorado (UL1 TR001082).

The authors report no conflicts of interest.

Supplemental digital content is available for this article. Direct URL citations appear in the printed text, and links to the digital files are provided in the HTML text of this article on the journal's Web site (www.jpgn.org).

Copyright $(102017$ by European Society for Pediatric Gastroenterology, Hepatology, and Nutrition and North American Society for Pediatric Gastroenterology, Hepatology, and Nutrition

DOI: 10.1097/MPG.0000000000001682 


\section{Exposure and Potential Influencing Factors}

Information about mode of delivery (vaginal delivery with or without instrumentation or C-section) was collected by questionnaires at enrollment, including information regarding premature rupture of membranes. Potential influencing factors for mode of delivery such as sociodemographic data and maternal and child characteristics were collected by questionnaires and included country of residence, maternal age and education, duration of gestation, birth weight and length, maternal age, maternal prepregnancy body mass index (BMI), gestational weight gain, smoking, antibiotic use, $\mathrm{T} 1 \mathrm{D}$, or any other diabetes during pregnancy and whether membranes ruptured before labor started. We categorized maternal education into lower (high school or less) and higher (college or university). Feeding variables, such as initiation and duration of exclusive breast-feeding, and timing of gluten introduction were assessed, as they are known to be influenced by mode of delivery (1). Hospitalization, diarrhea during the first 3 months of age, and antibiotic use in the infant during the first 3 and 12 months of life respectively were recorded. These post-partum factors and infant feeding patterns were considered as potential mediators through which C-section may have an indirect effect on the development of CDA and CD.

\section{Statistical Analysis}

Fisher exact test or Wilcoxon rank-sum test (as appropriate) was used to examine the associations of maternal and child characteristics with mode of delivery. Birth size was standardized per 40 weeks of gestational age (ie, birth weight [or length]/ gestational age in weeks*40 weeks]. A Cox proportional hazard regression model was used to examine the association between CDA or CD and mode of delivery. Time to CDA was defined as age when the first tTGA-positive blood sample was drawn, and the right-censored time was age when the last blood sample was collected for testing of tTGA. Time to diagnosis of CD was age at biopsy and the right-censored time was age of the last visit that was confirmed to be CD-free. For maternal and child characteristics, hazard ratios (HRs) and 95\% confidence intervals (CIs) were obtained after adjusting for country, sex, HLA risk group, and first-degree relative with $\mathrm{CD}$, as these factors were known to be associated with offspring's CD risk from previous studies (17). For mode of delivery, HRs with 95\% CI were investigated in 3 models: unadjusted, adjusted for the risk factors as listed above, and additionally adjusted for maternal education and duration of total breast-feeding, as these were found to be associated with CDA. Martingale residuals were used for the proportionality testing, and there was no evidence of violating the assumption on mode of delivery. As this analysis was a post hoc analysis, a sample size calculation was not performed. $P$ values $<0.05$ were considered to be statistically significant. All analyses were performed using SAS 9.4 (SAS Institute, Cary, NC).

\section{RESULTS}

\section{Participants and Mode of Delivery}

At the time of analysis, 6721 children were screened for tTGA in TEDDY (Fig. 1). Of those, 203 participants were excluded because they were either twins or triplets. Of the remaining 6518 singleton deliveries, $1600(25 \%)$ were born by C-section, 4487 $(69 \%)$ by normal vaginal delivery, $427(6 \%)$ with instrumental support (vacuum, forceps), and 4 children were missing the information. We analyzed 6087 children who were delivered by

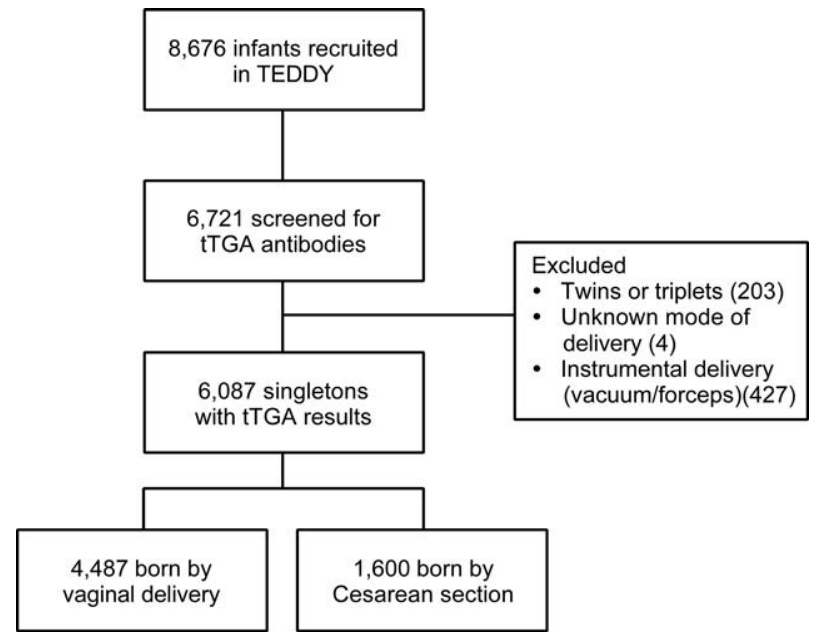

FIGURE 1. Flow chart of study population. TEDDY $=$ The Environmental Determinants for Diabetes in the Young; tTGA = tissue transglutaminase autoantibodies

C-section or normal vaginal delivery without an instrumental support. Of those children, 979 (16\%) had developed CDA and $343(6 \%)$ were diagnosed as having CD, of which $331(97 \%)$ were biopsy-proven. The median follow-up was 78 months (25th percentile $=63$ and 75 th percentile $=97$ ).

\section{Maternal and Child Characteristics in Relation to Mode of Delivery}

The C-section rates were highest in Germany (38\%), followed by the United States (37\%), Finland (18\%), and Sweden $(16 \%)$, respectively. Mothers giving birth by C-section had higher levels of education, were older, had a higher prepregnancy BMI, were more likely to have taken antibiotics, and were more likely to have a diabetic pregnancy compared to mothers giving birth by vaginal delivery (Table 1 ). Infants born by $\mathrm{C}$-section had a shorter gestational age than those children born by vaginal delivery. When the birth weight and length per 40 weeks of gestational age were considered, the body length was higher in infants born by $\mathrm{C}$-section, although median birth weight was almost equal in both groups (Table 1). When considering potential post-partum mediators for CDA or CD, infants born by C-section had a lower rate of initiation of breast-feeding, a shorter duration of exclusive and total breast-feeding, and a later introduction of gluten when compared to infants born by vaginal delivery (Table 1). Moreover, they showed a higher rate of diarrheal episodes during the first 3 months of life and a higher frequency of hospital admission and antibiotic use during the first year of life (Table 1).

\section{Known Risk Factors for Celiac Disease Autoimmunity and Celiac Disease in Relation to Mode of Delivery}

Country of birth was significantly associated with the mode of delivery. In contrast, HLA risk group (being homozygous for DQ2 vs heterozygous for DQ2 or DQ8 positive), female sex, and having a first-degree relative with $\mathrm{CD}$ (parent, sibling) were not 
$\overline{\text { TABLE 1. Maternal and child characteristics in relation to mode of delivery. For continuous variables, median (25th percentile, } 75 \text { th percentile) }}$ is reported

\begin{tabular}{|c|c|c|c|}
\hline & Vaginal delivery $(n=4487)$ & Cesarean delivery $(n=1600)$ & $P$ \\
\hline Age, y & $30(27,34)$ & $32(28,36)$ & $<0.0001$ \\
\hline$\leq$ High school & $838(19 \%)$ & $229(14 \%)$ & $<0.0001$ \\
\hline$>$ High school & $3551(79 \%)$ & $1331(83 \%)$ & \\
\hline Smoking during pregnancy & $498(11 \%)$ & $157(10 \%)$ & 0.19 \\
\hline Gestational weight gain, $\mathrm{kg}$ & $14(11,18)$ & $15(11,19)$ & 0.023 \\
\hline Any diabetes during pregnancy & $333(7 \%)$ & $276(17 \%)$ & $<0.0001$ \\
\hline \multicolumn{4}{|l|}{ Child characteristics } \\
\hline Gestational age, wk & $40(39,40.3)$ & $40(38,40)$ & $<0.0001$ \\
\hline Birth weight ( $\mathrm{kg} / 40$ weeks of gestational age) & $3.6(3.3,3.9)$ & $3.6(3.2,3.9)$ & 0.38 \\
\hline Birth length ( $\mathrm{cm} / 40$ weeks of gestational age) & $51(50,53)$ & $52(50,54)$ & $<0.0001$ \\
\hline$\leq 3 \mathrm{mo}$ & $81(4 \%)$ & $38(5 \%)$ & 0.567 \\
\hline $3-12 \mathrm{mo}$ & $1889(93 \%)$ & 749 (93\%) & \\
\hline$>12$ mo & $54(3 \%)$ & $18(2 \%)$ & $<0.0001$ \\
\hline Breast-feeding initiation & $4396(98 \%)$ & $1533(96 \%)$ & $<0.0001$ \\
\hline Duration of exclusive breast-feeding, days & $28(0.5,112)$ & $7(0.5,42)$ & $<0.0001$ \\
\hline Duration of total breast-feeding, wk & $35(17,52)$ & $30(8,50)$ & $<0.0001$ \\
\hline Age at gluten introduction, wk & $26(22,30)$ & $26(22,35)$ & \\
\hline \multicolumn{4}{|l|}{ Known risk factors for $\mathrm{CDA} / \mathrm{CD}$} \\
\hline \multicolumn{4}{|l|}{ HLA, n $(\%)$} \\
\hline DQ2/DQ2 & $889(20 \%)$ & $340(21 \%)$ & 0.22 \\
\hline Others & $3598(80 \%)$ & $1260(79 \%)$ & \\
\hline \multicolumn{4}{|l|}{ Sex, n (\%) } \\
\hline Girls & $2231(50 \%)$ & $761(48 \%)$ & 0.15 \\
\hline Sweden & $1580(35 \%)$ & $294(18 \%)$ & \\
\hline
\end{tabular}

$\mathrm{BMI}=$ body mass index $\mathrm{CD}=$ celiac disease $; \mathrm{CDA}=$ celiac disease autoimmunity.

significantly different between infants born by C-section and vaginally delivered children (Table 1).

\section{Maternal and Child Characteristics as Potential Risk Factors for Celiac Disease Autoimmunity and Celiac Disease}

Maternal and child characteristics proposed to be associated with the mode of delivery were further examined for association with CDA or CD development after adjustment for country, female sex, HLA-risk genotype, and first-degree relative status with CD (Table 2). Of those factors, only maternal education and total duration of breast-feeding were found to be associated with the risk of developing CDA. None of these factors was associated with the risk of developing $\mathrm{CD}$.

\section{Cesarean Section and Risk of Celiac Disease Autoimmunity and Celiac Disease in Offspring}

The percentages of children developing CDA was 17\% (754/ $4487)$ in the vaginally delivered children versus $14 \%(225 / 1600)$ in C-section $(P=0.03)$, the values for CD were 6\% (273/4487) versus 4\% (70/1600), respectively $(P=0.03)$.

Three models were used in the adjusted analysis of the effect of C-section on the development of CDA and CD (Table 3). First, in the crude model, children born by $\mathrm{C}$-section were less likely to develop CDA $(\mathrm{HR}=0.849 ; 95 \%$ CI $0.732,0.986 ; P=0.03)$ and $\mathrm{CD}(\mathrm{HR}=0.753 ; 95 \%$ CI $0.579,0.979 ; P=0.03)$, compared with vaginally delivered children (model 1 in Table 3 and Supplemental Digital Content, Figure, http://links.lww.com/MPG/B52). After adjusting for country, HLA-risk genotype, female sex, and first-degree relative status with $\mathrm{CD}$, the association of the 
TABLE 2. HR with $95 \% \mathrm{CI}$ for CD or CDA. For each characteristic, a Cox regression model was used after adjusting for country, sex, HLA, and firstdegree relatives with $C D$

CDA

HR $(95 \% \mathrm{CI}), P$
CD

HR $(95 \% \mathrm{CI}), P$

\footnotetext{
Maternal characteristics

Age, y

Education ( $>$ high school vs $\leq$ high school)

Antibiotic use (yes vs no)

Pre-pregnancy BMI, $\mathrm{kg} / \mathrm{m}^{2}$

Gestational weight gain, $\mathrm{kg}$

Any diabetes (yes vs no)

Child characteristics

Gestational age, wk

Birth length ( $\mathrm{cm} / 40 \mathrm{wk}$ of gestational age)

Hospitalized by 3 mo of age (yes vs no)

Diarrhea by 3 months old (yes vs no)

Antibiotic use (yes vs no)

Breast-feeding initiation (yes vs no)

Duration of exclusive breast-feeding, days

Duration of total breast-feeding, wk

Age at gluten introduction, wk
}

$1.002(0.990,1.015), 0.70$

$1.345(1.119,1.615), 0.002$

0.999 (0.851, 1.173), 0.99

$1.002(0.990,1.013), 0.79$

$0.993(0.982,1.003), 0.15$

$1.032(0.827,1.288), 0.78$

$1.035(0.992,1.080), 0.11$

$1.010(0.985,1.036), 0.44$

$0.987(0.819,1.190), 0.89$

$0.820(0.636,1.058), 0.13$

$1.096(0.961,1.249), 0.17$

$1.625(0.972,2.716), 0.06$

1.001 (1.000, 1.002), 0.05

1.003 (1.001, 1.004), 0.011

$1.006(0.997,1.014), 0.18$
$1.001(0.980,1.023), 0.91$

1.233 (0.920, 1.653), 0.16 $0.957(0.726,1.262), 0.76$ $0.995(0.974,1.016), 0.65$ 1.005 (0.988, 1.023), 0.56 $0.779(0.506,1.200), 0.26$

$1.040(0.967,1.119), 0.29$ $1.017(0.973,1.063), 0.45$ 0.962 ( $0.697,1.327), 0.81$ $0.901(0.594,1.366), 0.62$ $1.025(0.820,1.281), 0.83$ 1.027 (0.481, 2.190), 0.95 $1.002(1.000,1.003), 0.05$ 1.000 (0.997, 1.004), 0.83 $1.008(1.009,1.024), 0.24$

$\mathrm{CD}=$ celiac disease $\mathrm{CDA}=$ celiac disease autoimmunity $\mathrm{CI}=$ confidence interval; $\mathrm{HR}=$ hazard ratio.

mode of delivery with $\mathrm{CDA}$ or $\mathrm{CD}$ was no longer significant (model 2 in Table 3 and in Supplemental Digital Content, Figure, http://links.lww.com/MPG/B52). Furthermore, after adjustment for maternal education and total duration of breast-feeding (model 3 in Table 3 and in Supplemental Digital Content, Figure, http:// links.lww.com/MPG/B52), C-section delivery was not significantly associated with either CDA $(\mathrm{HR}=0.905 ; 95 \% \mathrm{CI}$ $0.775,1.056 ; P=0.20)$ or $\mathrm{CD}(\mathrm{HR}=0.850 ; 95 \%$ CI 0.649 , $1.113 ; P=0.24))$.
Of 1600 mothers with C-section, 149 (9.3\%) reported ruptured membranes before labor started. When we analyzed the development of CDA in children born by $\mathrm{C}$-section separately by premature rupture of membranes $(n=27)$, we did not find significant differences in the HRs compared to vaginally delivered infants before (crude HR 1.112; 95\% CI 0.757, 1.632; $P=0.59$ ) and after adjustment using model 2 (adj.HR 1.197; 95\% CI 0.813, 1.762; $P=0.36$ ) and model 3 (adj. HR 1.126; 95\%CI 0.760, 1.670; $P=0.55)$.

TABLE 3. HRs with $95 \%$ Cls for CD or CDA by mode of delivery (cesarean section), unadjusted (model 1) and adjusted for country, sex, HLA risk group, and first-degree relative with CD (model 2 ), and additionally for maternal education and breast-feeding duration (model 3 )

\begin{tabular}{|c|c|c|}
\hline & $\begin{array}{c}\text { CDA } \\
\text { HR }(95 \% \mathrm{CI}), P\end{array}$ & $\begin{array}{c}\text { CD } \\
\text { HR }(95 \% \mathrm{CI}), P\end{array}$ \\
\hline \multicolumn{3}{|l|}{ Model 1} \\
\hline C-section vs normal vaginal delivery & $0.849(0.732,0.986), 0.032$ & $0.753(0.579,0.979), 0.034$ \\
\hline \multicolumn{3}{|l|}{ Model 2} \\
\hline Country Finland vs United States & $1.152(0.966,1.373), 0.12$ & $1.026(0.742,1.420), 0.88$ \\
\hline Country Germany vs United States & $1.199(0.899,1.598), 0.22$ & $1.156(0.672,1.991), 0.60$ \\
\hline Country Sweden vs United States & $1.459(1.255,1.695),<0.0001$ & $1.893(1.468,2.440),<0.0001$ \\
\hline Girl vs boy & $1.538(1.354,1.746),<0.0001$ & $1.874(1.506,2.333),<0.0001$ \\
\hline HLA DR3/3 vs others & $3.398(2.986,3.866),<0.0001$ & $3.784(3.048,4.697),<0.0001$ \\
\hline Family history with CD vs not & $2.420(1.924,3.045),<0.0001$ & $3.935(2.901,5.336),<0.0001$ \\
\hline C-section vs normal vaginal delivery & $0.901(0.774,1.050), 0.18$ & $0.837(0.640,1.095), 0.19$ \\
\hline \multicolumn{3}{|l|}{ Model 3} \\
\hline Country Finland vs United States & $1.132(0.947,1.352), 0.17$ & $0.989(0.712,1.373), 0.95$ \\
\hline Country Germany vs United States & $1.178(0.873,1.591), 0.28$ & $1.151(0.657,2.015), 0.62$ \\
\hline Country Sweden vs United States & $1.553(1.329,1.814),<0.0001$ & $1.932(1.487,2.509),<0.0001$ \\
\hline Girl vs boy & $1.584(1.393,1.801),<0.0001$ & $1.923(1.542,2.399),<0.0001$ \\
\hline HLA DR3/3 vs others & $3.403(2.986,3.878),<0.0001$ & $3.749(3.015,4.661),<0.0001$ \\
\hline Family history with CD vs not & $2.381(1.889,3.001),<0.0001$ & $3.826(2.810,5.209),<0.0001$ \\
\hline Mother education $>$ high school vs $\leq$ high school & $1.332(1.106,1.604), 0.003$ & $1.263(0.937,1.702), 0.12$ \\
\hline Duration of total breast-feeding, wk & $1.002(1.000,1.004), 0.030$ & $1.000(0.996,1.004), 0.94$ \\
\hline $\mathrm{C}$-section vs normal vaginal delivery & $0.905(0.775,1.056), 0.20$ & $0.850(0.649,1.113), 0.24$ \\
\hline
\end{tabular}

$\mathrm{CD}=$ celiac disease $\mathrm{CDA}=$ celiac disease autoimmunity $\mathrm{CI}=$ confidence interval $\mathrm{HR}=$ hazard ratio. 


\section{DISCUSSION}

In TEDDY, one of the largest prospective screening studies for T1D and CD to date, mode of delivery was not an independent risk factor for the development of CDA or CD in children. We feel that this is the most definitive study to date on the role of C-section in development of CD. This finding disputes previous studies claiming that the mode of delivery might contribute toward development of CD (11-14) and supports others that have not found any association $(4,15,16,23)$. These differences may be because of the complex interactions and dependency between maternal/child characteristics, mode of delivery, post-partum feeding and drug use, and microbiome on the development of CDA and CD (Fig. 2). The TEDDY study, with its prospective design and multinational participation, allowed adjustment of these confounding factors associated with mode of delivery, as well as known factors associated with $\mathrm{CDA}$ and $\mathrm{CD}$, including genetics.

Firstly, this analysis included only newborns with known HLA-risk genotypes for $\mathrm{CD}$, which is a prerequisite for developing CDA and CD. HLA genotype greatly affects risk, and was adjusted for in the analysis (17). Other identified risk factors such as female sex, having a first-degree relative with $\mathrm{CD}$, and country of residence were adjusted for.

Second, almost $90 \%$ of children were recruited from the general population and were considered representative (whereas only $10.9 \%$ came from families with a first-degree relative with T1D). Only Germany selectively recruited for families with T1D (37\% of the German TEDDY participants), which was reflected in the higher percentage of mothers with T1D as compared with the other 3 countries $(18.6 \%$ vs $2.2 \%-3.7 \%$, respectively), and may explain in part the high $\mathrm{C}$-section rate in Germany. When excluding mothers with T1D the C-section rate in Germany decreased only marginally from $38 \%$ to $34 \%$ (data not shown) suggesting that the indication for C-section in Germany is mainly because of other reasons than maternal T1D. Moreover, we excluded twins, triplets, and infants born with instrumental intervention (vacuum, forceps) because they are more likely to have post-partum complications. We therefore feel confident that the TEDDY cohort can uniquely investigate this complex relationship between the $\mathrm{C}$-section and the risk for $\mathrm{CD}$ in the offspring (18).

Further strength of our study is the frequent longitudinal, active screening for $\mathrm{CDA}$ and $\mathrm{CD}$, allowing for timely determination of asymptomatic and subclinical cases of $\mathrm{CD}$, which represent the majority of children with $\mathrm{CD}$, even younger than 4 years $(21)$. Most previous studies used national birth and/or disease registries in which cases with $\mathrm{CD}$ were identified by pathology reporting or by their respective International Classification of Diseases (ICD) codes $(4,11,15)$. Another study used children with CD or inflammatory bowel disease from pediatric tertiary centers, with controls experiencing other diseases (12). Both study designs may be prone to selection bias because clinically detected patients with $\mathrm{CD}$ represent only a small part of all true cases with $\mathrm{CD}$ in the community $(24,25)$. In addition, mothers with TID or other autoimmune diseases are more likely to deliver by elective $\mathrm{C}$-section. T1D shares the same HLA risk alleles with CD, and children from diabetic mothers have a much higher risk to carry these HLA risk alleles compared to the general population. Lack of adjustment for the celiac risk alleles will result in a higher proportion of cases with $\mathrm{CD}$ in the $\mathrm{C}$-section group as a result of genetic predisposition, but not environmental factors. In our analysis, we did not only adjust for maternal TID but also for the different HLA risk alleles in cases and controls.

In concordance with others, we identified several maternal and child characteristics associated with delivery by C-section (26). Contrary to our hypothesis, C-section was initially inversely associated with CDA and CD in the crude analysis. After adjusting for 4 previously identified risk factors for CD in the TEDDY cohort (17), no association was found. Only 2 other factors remained significantly related to persistent autoimmunity in the Cox regression model: total duration of breast-feeding, as reported before (27) and

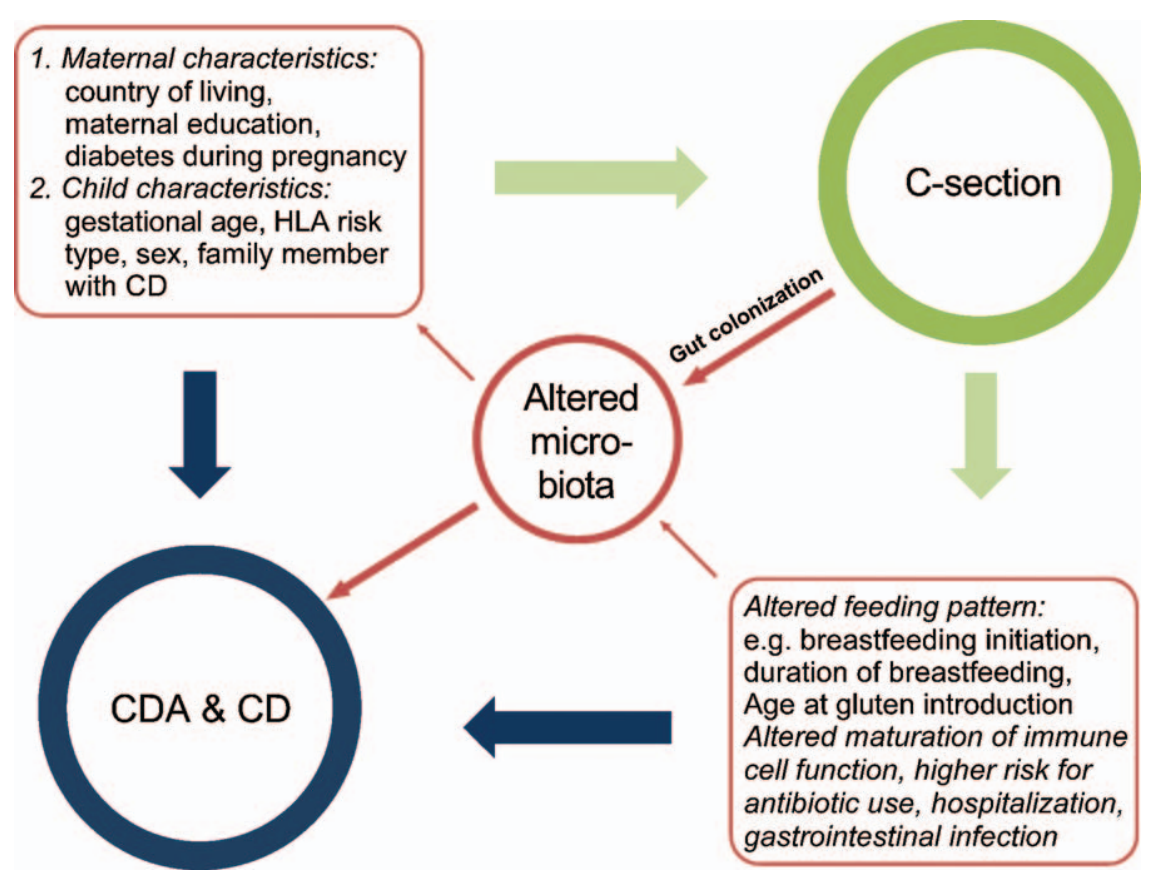

FIGURE 2. Complex interaction between maternal and child's characteristics, cesarean section and the possible consequences for post-partum factors, and the infant's microbiome on the risk of the development of CDA and CD. C-section $=$ cesarean section; $C D=$ celiac disease; $\mathrm{CDA}=$ celiac disease autoimmunity. 
a maternal education level above high school. Higher education level may be a proxy for a higher socioeconomic status and unmeasured environmental confounding factors. In previous studies using clinically detected cases with $\mathrm{CD}$, different health careseeking behavior of educated mothers and therefore a higher detection rate could introduce a bias. In this study, active screening independent of symptoms made that type of bias unlikely.

A limitation of our study is the lack of information regarding the type of C-section: emergency versus elective. Differences in total $\mathrm{C}$-section rates between countries are more likely because of planned than to intrapartum (emergency) C-section. Marild et al reported on pregnancy data from the Swedish Medical Birth Register between 1973 and 2008 and compared cases with villous atrophy with age- and sex-matched controls from the general population (14). They found a weak association between an elective cesarean delivery and $\mathrm{CD}$ in offspring (adjusted odds ratio $[\mathrm{OR}]=1.15)$, but no increased risk for $\mathrm{CD}$ after an emergency (adjusted $\mathrm{OR}=1.02$ ) or any $\mathrm{C}$-section (adjusted $\mathrm{OR}=1.06$ ). Kristensen et al reported the opposite from a population and national register-based cohort including all children born in Denmark from January 1997 through December 2012. Children delivered by emergency $\mathrm{C}$-section were at an increased risk for CD (adjusted $\mathrm{OR}=1.22$ ), whereas children delivered by elective C-section were not (adjusted OR $=0.69$ ). Several explanations have been proposed for the different outcomes in relation to type of C-section. Thyssen et al showed that the natural maturation of immune cells and the cord blood immune cell phenotypes are influenced by stress during vaginal delivery. This process is bypassed by elective $\mathrm{C}$-section, but not in-labor C-section (28). In emergency C-sections, the membranes are likely to be ruptured exposing the infant to the vaginal microbiome, whereas in elective C-section, this is not the case (14). A recent Danish study compared fecal microbiota pattern of infants born by emergency $\mathrm{C}$-section, by elective $\mathrm{C}$-section and by vaginal delivery at 3 time points (28). All infants in the $2 \mathrm{C}$-section groups were exposed to intrapartum antibiotics, but only $13 \%$ of naturally born infants. For most cultured bacterial strains, the difference at 1 week of age was less pronounced between the two $\mathrm{C}$-section groups compared to vaginally delivered neonates. The differences between the 3 groups were less pronounced at 1 month and had disappeared by 1 year of age. In our 149 cases reporting prematurely ruptured membranes, an emergency $\mathrm{C}$-section can be assumed with perinatal contact of the newborn with the maternal vaginal microbiota. We found no significantly different risk for later development of $\mathrm{CD}$ or CDA in subgroup analysis in those with prematurely ruptured membranes. A further limitation is that for ethical reasons we were not allowed to collect data on ethnicity in the European countries.

In conclusion, children do not have an increased risk for CDA or CD during childhood if they are born by C-section compared to vaginal delivery. Our cohort was prospectively screened for CDA and includes only children carrying the genetic risk alleles, which is a precondition to develop CD. One-quarter of the analyzed cohort was delivered by $\mathrm{C}$-section. After adjustment for potential confounders, we did not see even a trend for an increased risk of $\mathrm{CD}$ after $\mathrm{C}$-section. We suggest that environmental factors other than mode of delivery are more likely to be responsible for the increasing incidence of CD.

Acknowledgments: The authors express their gratitude to the children and parents who participated in the study, and the TEDDY staff in all centers for their excellent work over the Literature The Teddy Study Group information

Colorado Clinical Center: Marian Rewers, M.D., Ph.D., PI, ${ }^{1,4,5,6,10,11}$ Kimberly Bautista, ${ }^{12}$ Judith Baxter, ${ }^{9,10,12,15}$ Ruth Bedoy, ${ }^{2}$ Daniel Felipe-Morales, Brigitte I. Frohnert, M.D., ${ }^{2,14}$
Patricia Gesualdo, ${ }^{2,6,12,14,15}$ Michelle Hoffman, ${ }^{12-14}$ Rachel Karban, ${ }^{12}$ Edwin Liu, M.D., ${ }^{13}$ Jill Norris, Ph.D.,, ${ }^{2,12}$ Adela Samper-Imaz, Andrea Steck, M.D., ${ }^{3,14}$ Kathleen Waugh, ${ }^{6,7,12,15}$ Hali Wright. ${ }^{12}$ University of Colorado, Anschutz Medical Campus, Barbara Davis Center for Childhood Diabetes.

Georgia/Florida Clinical Center: Jin-Xiong She, Ph.D., PI, ${ }^{1,3,4,11}$ Desmond Schatz, M.D., ${ }^{* 4,5,7,8}$ Diane Hopkins, ${ }^{12}$ Leigh Steed, ${ }^{12-15}$ Jamie Thomas, ${ }^{* 6,12}$ Janey Adams, ${ }^{* 12}$ Katherine Silvis, ${ }^{2}$ Michael Haller, M.D., ${ }^{* 14}$ Melissa Gardiner, Richard McIndoe, Ph.D., Ashok Sharma, Joshua Williams, Gabriela Young, Stephen W. Anderson, M.D., ${ }^{\wedge}$ Laura Jacobsen, M.D. ${ }^{* 14}$ Center for Biotechnology and Genomic Medicine, Augusta University. *University of Florida, ${ }^{\wedge}$ Paediatric Endocrine Associates, Atlanta.

Germany Clinical Center: Anette G. Ziegler, M.D., PI, ${ }^{1,3,4,11}$ Andreas Beyerlein, Ph.D., ${ }^{2}$ Ezio Bonifacio Ph.D., ${ }^{* 5}$ Michael Hummel, M.D., ${ }^{13}$ Sandra Hummel, Ph.D., ${ }^{2}$ Kristina Foterek, ${ }^{¥ 2}$ Nicole Janz, Mathilde Kersting, Ph.D., ${ }^{\ngtr 2}$ Annette Knopff, ${ }^{7}$ Sibylle Koletzko, M.D., ${ }^{\top 13}$ Claudia Peplow, ${ }^{12}$ Roswith Roth, Ph.D., Marlon Scholz, Joanna Stock, ${ }^{9,12}$ Elisabeth Strauss, ${ }^{12,14}$ Katharina Warncke, M.D., ${ }^{14}$ Lorena Wendel, Christiane Winkler, Ph.D. ${ }^{2,12,15}$ Forschergruppe Diabetes e.V. and Institute of Diabetes Research, Helmholtz Zentrum München, and Klinikum rechts der Isar, Technische Universität München. * Center for Regenerative Therapies, TU Dresden, “Dr. von Hauner Children's Hospital, Department of Gastroenterology, Ludwig Maximillians University Munich, ${ }^{¥}$ Research Institute for Child Nutrition, Dortmund.

Finland Clinical Center: Jorma Toppari, M.D., Ph.D., PI ${ }^{¥ \wedge} 1,4,11,14$ Olli G. Simell, M.D., Ph.D., ${ }^{\wedge} 1,4,11,13$ Annika Adamsson, Ph.D., ${ }^{\wedge 12}$ Suvi Ahonen, ${ }^{* \pm \S}$ Heikki Hyöty, M.D., Ph.D., ${ }^{* \pm 6}$ Jorma Ilonen, M.D., Ph.D., ${ }^{¥} \uparrow^{3}$ Sanna Jokipuu, ${ }^{\wedge}$ Tiina Kallio, ${ }^{\wedge}$ Leena Karlsson, ${ }^{\wedge}$ Miia Kähönen, ${ }^{\mu} \propto$ Mikael Knip, M.D., Ph.D., ${ }^{* \pm 5}$ Lea Kovanen, ${ }^{* \pm \S}$ Mirva Koreasalo, ${ }^{* \pm 2}$ Kalle Kurppa, M.D., Ph.D., ${ }^{* \pm 13}$ Tiina Latvaaho, ${ }^{\mu \alpha}$ Maria Lönnrot, M.D., Ph.D., ${ }^{* \pm 6}$ Elina Mäntymäki, ${ }^{\wedge}$ Katja Multasuo, ${ }^{\mu} \propto$ Juha Mykkänen, Ph.D., ${ }^{3}$ Tiina Niininen, ${ }^{ \pm * 12}$ Sari Niinistö, ${ }^{ \pm \S}$ Mia Nyblom, ${ }^{* \pm}$ Petra Rajala, Jenna Rautanen, ${ }^{ \pm \S}$ Anne Riikonen, ${ }^{* \pm \S}$ Mika Riikonen, ${ }^{\wedge}$ Jenni Rouhiainen, ${ }^{\wedge}$ Minna Romo, Tuula Simell, Ph.D., Ville Simell, ${ }^{\wedge 13}$ Maija Sjöberg, ${ }^{\ddagger^{\wedge} 12,14}$ Aino Stenius, ${ }^{\mu \ltimes 12}$ Maria Leppänen, ${ }^{\wedge}$ Sini Vainionpää, ${ }^{\wedge}$ Eeva Varjonen, ${ }^{\sharp \wedge 12}$ Riitta Veijola, M.D., Ph.D., ${ }^{\mu \ltimes 14}$ Suvi M. Virtanen, M.D., Ph.D., ${ }^{* \pm \$ 2}$ Mari Vähä-Mäkilä, ${ }^{\wedge}$ Mari Åkerlund, ${ }^{* \pm \S}$ Katri Lindfors, Ph.D. ${ }^{* 13}$ ${ }^{¥}$ University of Turku, ${ }^{*}$ University of Tampere, ${ }^{\mu}$ University of Oulu, ^Turku University Hospital, Hospital District of Southwest Finland, ${ }^{ \pm}$Tampere University Hospital, ${ }^{\infty}$ Oulu University Hospital, ${ }^{\S}$ National Institute for Health and Welfare, Finland, ${ }^{\text {University }}$ of Kuopio.

Sweden Clinical Center: Åke Lernmark, Ph.D., PI, 1,3-6,8,10,11,15 Daniel Agardh, M.D., Ph.D., ${ }^{13}$ Carin Andrén Aronsson, ${ }^{2,13}$ Maria Ask, Jenny Bremer, Ulla-Marie Carlsson, Corrado Cilio, Ph.D., M.D. ${ }^{5}$ Emelie Ericson-Hallström, Lina Fransson, Thomas Gard, Joanna Gerardsson, Rasmus Bennet, Monica Hansen, Gertie Hansson, ${ }^{12}$ Cecilia Harmby, Susanne Hyberg, Fredrik Johansen, Berglind Jonsdottir M.D., Helena Elding Larsson M.D., Ph.D., ${ }^{6,14}$ Sigrid Lenrick Forss, Markus Lundgren, ${ }^{14}$ Maria Månsson-Martinez, Maria Markan, Jessica Melin, ${ }^{12}$ Zeliha Mestan, Kobra Rahmati, Anita Ramelius, Anna Rosenquist, Falastin Salami, Sara Sibthorpe, Birgitta Sjöberg, Ulrica Swartling, Ph.D., ${ }^{9,12}$ Evelyn Tekum Amboh, Carina Törn, Ph.D., ${ }^{3,15}$ Anne Wallin, Asa Wimar, ${ }^{12,14}$ Sofie Åberg. Lund University.

Washington Clinical Center: William A. Hagopian, M.D., Ph.D., PI, ${ }^{1,3,4-7,11,13,14}$ Michael Killian, ${ }^{6,7,12,13}$ Claire Cowen Crouch, ${ }^{12,14,15}$ Jennifer Skidmore, ${ }^{2}$ Josephine Carson, Kayleen Dunson, Rachel Hervey, Corbin Johnson, Rachel Lyons, Arlene Meyer, Denise Mulenga, Allison Schwartz, Joshua Stabbert, Alexander Tarr, Morgan Uland, John Willis. Pacific Northwest Diabetes Research Institute. 
Pennsylvania Satellite Center: Dorothy Becker, M.D., Margaret Franciscus, MaryEllen Dalmagro-Elias Smith, ${ }^{2}$ Ashi Daftary, M.D., Mary Beth Klein, Chrystal Yates. Children's Hospital of Pittsburgh of UPMC.

Data Coordinating Center: Jeffrey P. Krischer, Ph.D., PI, ${ }^{1,4,5,10,11}$ Michael Abbondondolo, Sarah Austin-Gonzalez, Maryouri Avendano, Sandra Baethke, Rasheedah Brown, ${ }^{12,15}$ Brant Burkhardt, Ph.D., ${ }^{5,6}$ Martha Butterworth, ${ }^{2}$ Joanna Clasen, David Cuthbertson, Christopher Eberhard, Steven Fiske, ${ }^{9}$ Dena Garcia, Jennifer Garmeson, Veena Gowda, Kathleen Heyman, Francisco Perez Laras, Hye-Seung Lee, Ph.D., ,2,13,15 Shu Liu, Xiang Liu, Ph.D., 2,3,9,14 Kristian Lynch, Ph.D., ${ }^{5,6,9,15}$ Jamie Malloy, Cristina McCarthy, ${ }^{12,15}$ Steven Meulemans, Hemang Parikh, Ph.D., ${ }^{3}$ Chris Shaffer, Laura Smith, Ph.D., ${ }^{9,12}$ Susan Smith, ${ }^{12,15}$ Noah Sulman, Ph.D., Roy Tamura, Ph.D., ,',2,13 Ulla Uusitalo, Ph.D., ${ }^{2,15}$ Kendra Vehik, Ph.D., ${ }^{4-6,14,15}$ Ponni Vijayakandipan, Keith Wood, Jimin Yang, Ph.D., R.D. ${ }^{2,15}$ Past staff: Lori Ballard, David Hadley, Ph.D., Wendy McLeod. University of South Florida.

Project scientist: Beena Akolkar, Ph.D. ${ }^{1,3,4-7,10,11}$ National Institutes of Diabetes and Digestive and Kidney Diseases.

Other contributors: Kasia Bourcier, Ph.D., ${ }^{5}$ National Institutes of Allergy and Infectious Diseases. Thomas Briese, Ph.D., ${ }^{6,15}$ Columbia University. Suzanne Bennett Johnson, Ph.D., ${ }^{9,12}$ Florida State University. Eric Triplett, Ph.D., ${ }^{6}$ University of Florida.

Committees:

${ }^{1}$ Ancillary Studies, ${ }^{2}$ Diet, ${ }^{3}$ Genetics, ${ }^{4}$ Human Subjects/Publicity/ Publications, ${ }^{5}$ Immune Markers, ${ }^{6}$ Infectious Agents, ${ }^{7}$ Laboratory Implementation, ${ }^{8}$ Maternal Studies, ${ }^{9}$ Psychosocial, ${ }^{10}$ Quality Assurance, ${ }^{11}$ Steering, ${ }^{12}$ Study Coordinators, ${ }^{13}$ Celiac Disease, ${ }^{14} \mathrm{Clin}-$ ical Implementation, ${ }^{15}$ Quality Assurance Subcommittee on Data Quality.

\section{REFERENCES}

1. Hyde MJ, Mostyn A, Modi N, et al. The health implications of birth by Caesarean section. Biol Rev Camb Philos Soc 2012;87:229-43.

2. Thavagnanam S, Fleming J, Bromley A, et al. A meta-analysis of the association between caesarean section and childhood asthma. Clin Exp Allergy 2008;38:629-33.

3. Cardwell CR, Stene LC, Joner G, et al. Caesarean section is associated with an increased risk of childhood-onset type 1 diabetes mellitus: a meta-analysis of observational studies. Diabetologia 2008;51:726-35.

4. Sevelsted A, Stokholm J, Bonnelykke K, et al. Cesarean section and chronic immune disorders. Pediatrics 2015;135:e92-8.

5. McLean MH, Dieguez D Jr, Miller LM, et al. Does the microbiota play a role in the pathogenesis of autoimmune diseases? Gut 2015;64:332-41.

6. Dominguez-Bello MG, Costello EK, Contreras M, et al. Delivery mode shapes the acquisition and structure of the initial microbiota across multiple body habitats in newborns. Proc Natl Acad Sci U S A 2010;107: 11971-5.

7. Husby S, Koletzko S, Korponay-Szabo IR, et al. European Society for Pediatric Gastroenterology, Hepatology, and Nutrition guidelines for the diagnosis of coeliac disease. J Pediatr Gastroenterol Nutr 2012;54: 136-60.

8. Lohi S, Mustalahti K, Kaukinen K, et al. Increasing prevalence of coeliac disease over time. Aliment Pharmacol Ther 2007;26:1217-25.
9. Gutierrez-Achury J, Zhernakova A, Pulit SL, et al. Fine mapping in the MHC region accounts for $18 \%$ additional genetic risk for celiac disease. Nat Genet 2015;47:577-8.

10. Rubio-Tapia A, Kyle RA, Kaplan EL, et al. Increased prevalence and mortality in undiagnosed celiac disease. Gastroenterology 2009;137: 88-93.

11. Kristensen K, Henriksen L. Cesarean section and disease associated with immune function. J Allergy Clin Immunol 2016;137:587-90.

12. Decker E, Engelmann G, Findeisen A, et al. Cesarean delivery is associated with celiac disease but not inflammatory bowel disease in children. Pediatrics 2010;125:e1433-40.

13. Adlercreutz EH, Wingren CJ, Vincente RP, et al. Perinatal risk factors increase the risk of being affected by both type 1 diabetes and coeliac disease. Acta Paediatr 2015;104:178-84.

14. Marild K, Stephansson O, Montgomery S, et al. Pregnancy outcome and risk of celiac disease in offspring: a nationwide case-control study. Gastroenterology 2012;142:39-45.

15. Roberts SE, Williams JG, Meddings D, et al. Perinatal risk factors and coeliac disease in children and young adults: a record linkage study. Aliment Pharmacol Ther 2009;29:222-31.

16. Emilsson L, Magnus MC, Stordal K. Perinatal risk factors for development of celiac disease in children, based on the prospective norwegian mother and child cohort study. Clin Gastroenterol Hepatol 2015;13:921-7.

17. Liu E, Lee HS, Aronsson CA, et al. Risk of pediatric celiac disease according to HLA haplotype and country. N Engl J Med 2014;371: $42-9$.

18. The Environmental Determinants of Diabetes in the Young (TEDDY) study: study design. Pediatr Diabetes 2007;8:286-98.

19. Hagopian WA, Erlich H, Lernmark A, et al. The Environmental Determinants of Diabetes in the Young (TEDDY): genetic criteria and international diabetes risk screening of 421000 infants. Pediatr Diabetes 2011;12:733-43.

20. Vehik K, Fiske SW, Logan CA, et al. Methods, quality control and specimen management in an international multicentre investigation of type 1 diabetes: TEDDY. Diabetes Metab Res Rev 2013;29: $557-67$.

21. Agardh D, Lee HS, Kurppa K, et al. Clinical features of celiac disease: a prospective birth cohort. Pediatrics 2015;135:627-34.

22. Oberhuber G, Granditsch G, Vogelsang H. The histopathology of coeliac disease: time for a standardized report scheme for pathologists. Eur J Gastroenterol Hepatol 1999;11:1185-94.

23. Lionetti E, Castellaneta S, Francavilla R, et al. Mode of delivery and risk of celiac disease: risk of celiac disease and age at gluten introduction cohort study. J Pediatr 2017;184:81-86.e2.

24. Laass MW, Schmitz R, Uhlig HH, et al. The prevalence of celiac disease in children and adolescents in Germany. Dtsch Arztebl Int 2015;112: $553-60$.

25. Myleus A, Ivarsson A, Webb C, et al. Celiac disease revealed in 3\% of Swedish 12-year-olds born during an epidemic. J Pediatr Gastroenterol Nutr 2009;49:170-6.

26. Laubereau B, Filipiak-Pittroff B, von Berg A, et al. Caesarean section and gastrointestinal symptoms, atopic dermatitis, and sensitisation during the first year of life. Arch Dis Child 2004;89:993-7.

27. Aronsson CA, Lee HS, Liu E, et al. Age at gluten introduction and risk of celiac disease. Pediatrics 2015;135:239-45.

28. Thysen AH, Larsen JM, Rasmussen MA, et al. Prelabor cesarean section bypasses natural immune cell maturation. J Allergy Clin Immunol 2015;136:1123-5. 